\title{
Application of Laser-Enhanced Ionization to Flame Temperature Determination
}

\author{
KING-DOW SU, CHUNG-YUAN CHEN, KING-CHUEN LIN, ${ }^{*}$ and WEI-TZOU LUH \\ Department of Chemistry, National Taiwan University and Institute of Atomic and Molecular Sciences, Academia Sinica, \\ P.O. Box 23-166, Taipei, Taiwan 10764, Republic of China (K.-D.S., C.-Y.C., K.-C.L.); and Institute of Atomic and Molecular \\ Sciences, Academia Sinica, P.O. Box 23-166, Taipei, Taiwan 10764, Republic of China (W.-T.L.)
}

\begin{abstract}
We have both theoretically and experimentally demonstrated that the laser-enhanced ionization (LEI) technique can be a simple, alternative method for flame temperature determination in an atmospheric air/acetylene flame. A three-level model of rate equations is developed to determine a flame temperature based upon the Boltzmann population of the ground fine structures of the analyte selected. A Ga sample was used for the test, and the LEI signal was measured with excitation to various intermediate states. The determined flame temperatures are very consistent with each other and also with those previously reported.

Index Headings: Atomic absorption spectroscopy; Flame spectroscopy.
\end{abstract}

\section{INTRODUCTION}

Temperature measurement has been an important subject in the area of combustion chemistry. The methods used for the temperature determination in a flame have been explored mostly by using various optical spectroscopies, such as the line reversal method, the two-line method, laser-induced fluorescence, Raman spectroscopy, and Rayleigh scattering. ${ }^{1,2}$ Most of these approaches rely upon the assumption of local thermal equilibrium, in which the flame temperature is represented in terms of electronic, vibrational, rotational, or translational behaviors.

Laser-enhanced ionization (LEI) has been developed as a powerful tool for detecting trace metals to the sub$\mathrm{pg} / \mathrm{mL}$ level in aqueous solution with extremely high sensitivity and selectivity. ${ }^{3-9} \mathrm{As}$ an alternative to the conventional UV detection, LEI has been successfully coupled to liquid chromatography as a detector. ${ }^{10,11}$ In addition, it has been employed to monitor the population density of species released ${ }^{12,13}$ and to determine the diffusion and mobility coefficients associated in a flame. ${ }^{14,15}$ In terms of these coefficients, the flame temperature has been estimated according to the Einstein relation..$^{15}$ As is the case for most methods, the temperature thus obtained is based on the assumption of local thermal equilibrium. The advantages in the use of LEI lie in its capability of achieving a very high spatial resolution and avoiding the effects of optical interference. Nevertheless, the inherent systematic error seems to be large, since a homogeneous electric field between two probing electrodes has to be assumed and the electrode dimensions and the convection velocity are also neglected. ${ }^{15}$

In this paper, instead of following the Einstein relation, we have derived a theoretical model to yield the flame temperature based upon the Boltzmann popula-

\footnotetext{
Received 17 May 1991.

* Author to whom correspondence should be sent.
}

tion of the ground fine structures of the species selected. A Ga sample has been used for the test; the resultant temperatures under different intermediate states are consistent with each other and also lie in the acceptable region when compared with those achieved by other techniques reported previously. In the following, a brief theoretical overview is first presented, the experimental details are then described and, finally, the results are presented.

\section{THEORY}

To deal with the laser-enhanced ionization (LEI) phenomena of atoms in flame, one should consider both the atomic $(l \rightarrow u)$ transition and subsequent $(u \rightarrow i)$ ionization process. ${ }^{1,6,16}$ The schematic diagram for atomic levels discussed in this work is shown in Fig. 1.

It has been shown previously by Omenetto et al. ${ }^{6}$ and Travis $^{16}$ that the ionization signal is proportional to the product of the steady-state population density, $n_{u}$, of the excited energy level, $u$, and its ionization rate coefficient, $K_{u i}$, due to collision.

Within the limit of the two-level approximation and with the assumption of a broad-band laser, $\dagger$ the population density, $n_{u}$, would be increased due to optical absorption $\left[n_{l}\left(g_{u} / g_{l}\right) A_{u l} \rho_{\lambda} \lambda_{u l}^{5} /(8 \pi h c)\right]$ and collisional excitation $\left[n_{l} K_{l}\right]$ from the lower level $l$, and also be decreased due to spontaneous emission $\left(n_{u} A_{u l}\right)$, induced emission $\left[n_{u} A_{u l} \rho_{\lambda} \lambda_{u l}^{5} /(8 \pi h c)\right]$, and collisional decay $\left(n_{u} K_{u}\right)$ to the lower level $l .{ }^{1}$ The steady-state population density $n_{u}$ then follows the relation

$$
\begin{aligned}
n_{l}\left(g_{u} / g_{l}\right) A_{u l} \rho_{\lambda} \lambda_{u l}^{5} /(8 \pi h c)+n_{l} K_{l} & =n_{u} A_{u l}+n_{u} A_{u l} \rho_{\lambda} \lambda_{u l}^{5} /(8 \pi h c)+n_{u} K_{u}
\end{aligned}
$$

where $n_{l}$ is the population density of the lower level $l$; $A_{u l}$ the spontaneous transition probability of the upper level $u ; \rho_{\lambda}$ the spectral density at the transition wavelength $\lambda_{u} ;, h$ the Planck's constant, $c$ the speed of light; $K_{l}$ the collision excitation rate coefficient; and $K_{u}$ the collision decay rate coefficient. The collision excitation term $n_{l} K_{l}$ can be neglected since the energy of the upper level $u$ concerned in this work is at least $3 \mathrm{eV}$ higher than the lower level $l .^{1}$ If the following four terms- $\tau_{u}=\left(A_{u l}\right.$ $\left.+K_{u}\right)^{-1}$, the lifetime of the upper level $u ; n_{t}=n_{u}+n_{l}$, the total number density of the interested atom; the

$\dagger$ The typical Doppler linewidth for the atomic transitions involved in this work is $1-5 \mathrm{GHz}$, which is smaller than the dye laser linewidth of $6-12 \mathrm{GHz}$ 


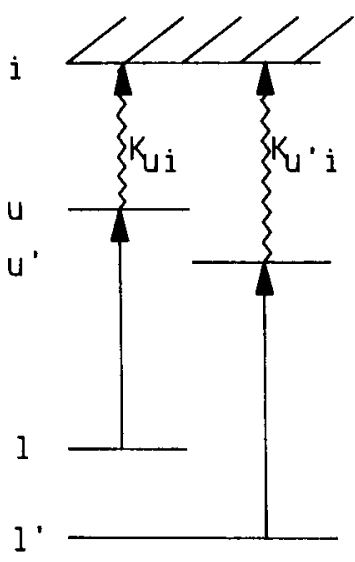

(a)

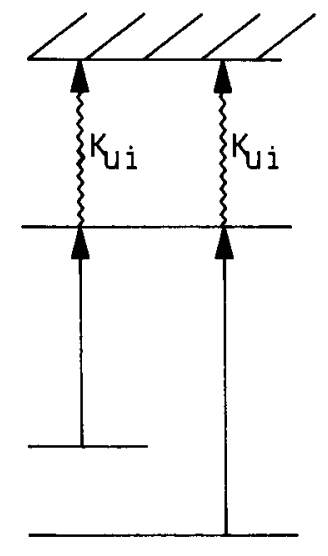

(b) i

u

1

$1^{\prime}$

FIG. 1. Schematic representations for two possible excitation schemes: (a) $\left[l^{\prime} \rightarrow u^{\prime} ; l \rightarrow u\right]$ and (b) $\left[l^{\prime} \rightarrow u ; l \rightarrow u\right]$.

spectral irradiance $I_{\lambda}=\rho_{\lambda} c$; and its saturation parameter $I_{\lambda}^{\mathrm{s}}=8 \pi h c^{2} g_{l} /\left(g_{u}+g_{l}\right) \tau_{u} A_{u l} \lambda_{u l}^{5}$-are assumed, then $n_{u}$ and $n_{l}$ can be derived as ${ }^{1,6}$

$$
\begin{aligned}
n_{u} & =n_{t} g_{u} I_{\lambda} /\left(g_{u}+g_{l}\right)\left(I_{\lambda}^{\mathrm{s}}+I_{\lambda}\right), \\
n_{l} & =n_{t}\left(\left(g_{u}+g_{l}\right) I_{\lambda}^{\mathrm{s}}+I_{\lambda}\right) /\left(g_{u}+g_{l}\right)\left(I_{\lambda}^{\mathrm{s}}+I_{\lambda}\right) .
\end{aligned}
$$

If a very weak laser intensity, $I_{\lambda} \ll I_{\lambda}^{\mathrm{s}}$, is assumed, then $n_{l}$ is close to its thermal number density $n_{l}^{\text {th }}$, and $n_{u}$ can then be approximated as

$$
n_{u} \simeq n_{l}^{\mathrm{th}}\left(g_{u} / g_{l}\right) I_{\lambda} \lambda_{u l}^{5} A_{u l} \tau_{u} /\left(8 \pi h c^{2}\right) .
$$

If a fast collision redistribution among excited levels lying between the excited level $u$ and the continuum state $i$ is assumed, then the ionization rate coefficient $K_{u i}$, due to collision, for the excited level $u$ may be given as ${ }^{6}$

$$
K_{u i}=\bar{v} \sigma_{u} n_{j} \exp \left[-\left(E_{i}-E_{u}\right) / k T\right],
$$

where $\bar{v}$ is the mean relative velocity between the atom and the colliding species $j$; $\sigma_{u}$ the cross section for the colliding process; $n_{j}$ the number density of the colliding species $j ; E_{i}$ the energy of the continuum state $i ; E_{u}$ the energy of the upper level $u ; k$ the Boltzmann constant; and $T$ the flame temperature. Thus the LEI signal $S_{u l}$, out of the upper level $u$ which was optically excited from the lower level $l$, can be described as

$$
S_{u l} \alpha n_{l}^{\mathrm{th}}\left(g_{u} / g_{l}\right) I_{\lambda} \lambda_{u l}^{5} A_{u l} \tau_{u} \sigma_{u} \exp \left[-\left(E_{i}-E_{u}\right) / k T\right] .
$$

Since we are interested in applying the LEI technique for flame temperature measurements, at least two lower thermally populated levels have to be considered. For the simplest situation, two lower levels $l$ and $l^{\prime}$, there are two possible excitation schemes, $\left(l^{\prime} \rightarrow u^{\prime}, l \rightarrow u\right)$ and $\left(l^{\prime}\right.$ $\rightarrow u, l \rightarrow u$ ), as shown in Fig. 1, available for our purpose. Here it is also assumed that the energy of the level $l$ is higher than that of the level $l^{\prime}$, and since the thermal number density of each lower level follows the Boltzmann distribution, we arrive at this useful relation:

$$
n_{l}^{\text {th }} / n_{l}^{\text {th' }}=\left(g_{l} / g_{l}\right) \exp \left[-\left(E_{l}-E_{l}\right) / k T\right] .
$$

For the $\left(l^{\prime} \rightarrow u^{\prime}, l \rightarrow u\right)$ excitation scheme, assuming that the laser intensity is very weak, using the relations in Eqs. 4, 6, and 7, we can derive the ratio of two LEI signals, $S_{u l} / S_{u^{\prime} l}$, as

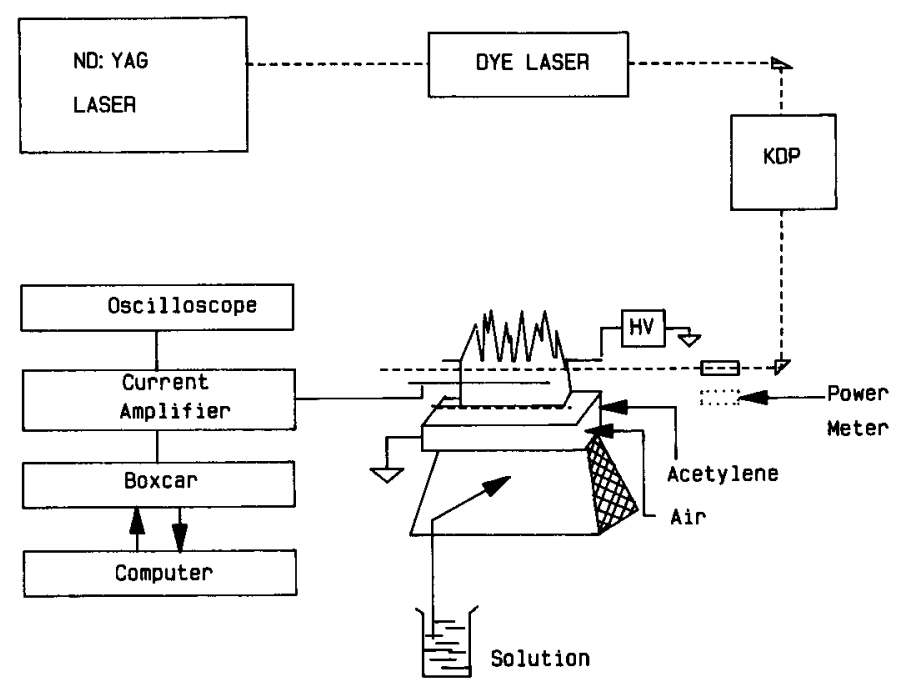

FIG. 2. Experimental setup for laser enhanced ionization in flame.

$$
\begin{aligned}
S_{u l} / S_{u^{\prime} l^{\prime}}= & \left(I_{\lambda} / I_{\lambda}^{\prime}\right)\left(\lambda_{u l}^{5} / \lambda_{u^{\prime} l^{\prime}}^{5}\right)\left(A_{u l} \tau_{u^{\prime}} / A_{u^{\prime} l^{\prime}} \tau_{u^{\prime}}\right)\left(g_{u} / g_{u^{\prime}}\right)\left(\sigma_{u} / \sigma_{u^{\prime}}\right) \\
& \times \exp \left[\left(E_{u}-E_{u^{\prime}}-E_{l}+E_{l^{\prime}}\right) / k T\right] .
\end{aligned}
$$

This equation can be further rearranged to give an explicit, practical relation for the temperature measurement in this work as

$$
\begin{aligned}
& T=\left(E_{u}-E_{u^{\prime}}-E_{l}+E_{l^{\prime}}\right) /(k Q), \\
& Q=\ln \frac{S_{u l} I_{\lambda}^{\prime} \lambda_{u^{\prime} l^{\prime}}^{5} A_{u^{\prime} l^{\prime}} \tau_{u^{\prime}} g_{u^{\prime}} \sigma_{u^{\prime}}}{S_{u^{\prime} l^{\prime}} I_{\lambda} \lambda_{u l}^{5} A_{u l} \tau_{u} g_{u} \sigma_{u}} .
\end{aligned}
$$

For the $\left(l^{\prime} \rightarrow u, l \rightarrow u\right)$ excitation scheme, we find a simpler relation as

$$
\begin{aligned}
T & \left.=\left(E_{l^{\prime}}-E_{l}\right) / k Q^{\prime}\right), \\
Q^{\prime} & =\ln \left(S_{u l} I_{\lambda}^{\prime} \lambda_{u l}^{5} A_{u l} / S_{u l} I_{\lambda} \lambda_{u l}^{5} A_{u l}\right) .
\end{aligned}
$$

Thus it is obvious that, if the spontaneous transition probabilities $A_{u l}$ and corresponding transition wavelengths $\lambda_{u l}$ for some pertinent atomic transitions $(l \rightarrow u)$ of an atom occurring in flame are available from the known literature, ${ }^{17}$ then by measuring the LEI signal of this atom and the power dependence of the LEI signal [i.e., $\left(S_{u l} / I_{\lambda}\right)$ ], one may then determine the flame temperature by using Eq. 9 for the scheme $\left(l^{\prime} \rightarrow u^{\prime} ; l \rightarrow u\right)$, provided that relevant lifetimes $\tau$ and cross sections $\sigma$ of level $u$ and $u^{\prime}$ are also known, or by using Eq. 10 for the scheme $\left(l^{\prime} \rightarrow u ; l \rightarrow u\right)$.

\section{EXPERIMENTAL}

The experimental setup for the LEI experiment is shown in Fig. 2. Since it was previously detailed, ${ }^{3,8}$ only a brief account is given below.

We performed the LEI experiment in an atmospheric flame. We used a commercial burner assembly (PerkinElmer) with a $100-\mathrm{mm} \times 0.5-\mathrm{mm}$ slot burner head, coupled with an interlocked gas control system by which acetylene $(0.5 \mathrm{~L} / \mathrm{min})$ and air $(12.5 \mathrm{~L} / \mathrm{min})$ were premixed prior to reaching the burner head. The aqueous solution of the Ga salt was prepared at $100 \mathrm{ppm}$, and was then aspirated at a flow rate of $4.5 \mathrm{cc} / \mathrm{min}$ into the burner head.

In this work, a tunable dye laser (Quanta Ray PDL- 
TABLE I. Atomic transitions and relevant spectral constants ${ }^{17}$ of the Ga element. $\lambda$ and $A$ represent the transition wavelength and the transition probability of each atomic transition, respectively. The uncertainty for the $A$ value is $25 \%$.

\begin{tabular}{clcc}
\hline Element & Transition & $\lambda(\mathrm{nm})$ & $A\left(10^{8} \mathrm{~s}^{-1}\right)$ \\
\hline $\mathrm{Ga}$ & $4^{2} P_{1 / 2} \rightarrow 5^{2} S_{1 / 2}$ & 403.298 & 0.49 \\
& $4^{2} P_{3 / 2} \rightarrow 5^{2} S_{1 / 2}$ & 417.227 & 0.92 \\
& $4^{2} P_{1 / 2} \rightarrow 4^{2} D_{3 / 2}$ & 287.465 & 1.2 \\
& $4^{2} P_{3 / 2} \rightarrow 4^{2} D_{3 / 2}$ & 294.480 & 0.27 \\
\hline
\end{tabular}

2), pumped by a frequency-doubled Nd:YAG laser (DCR2A), was used. As listed in Table I, the dye laser output could be directly used or be further frequency doubled with a KDP crystal housed in a wavelength extender (WEX-1), depending on the atom transitions of interest. Five different laser dyes, including Rhodamine 590, Rhodamine 610, DCM, Kiton red, and LDS 698 (all from Exciton), were used in the work. The dye laser had pulsed duration of approximately $5-8 \mathrm{~ns}$, and its output power, varying in the range of $20 \mu \mathrm{J}$ to $30 \mathrm{~mJ}$, was monitored by a surface absorbing disc calorimeter (Scientech 360001) throughout the experiment. The laser beam was directed longitudinally through the flame at $7.5 \pm 0.5$ $\mathrm{mm}$ from the burner head.

The enhanced ionization was detected by a pair of 100 $\mathrm{mm}$-long nichrome electrodes along the laser beam. The two electrodes were positioned $12 \pm 1 \mathrm{~mm}$ apart and biased at a voltage $500 \mathrm{~V}$. The LEI current signal was amplified with a current amplifier (Keithley 427) before feeding into a boxcar averager system (PAR 4400) for signal processing. The resulting output was then stored for later data treatment.

To confirm the atomic transitions of interest, we also acquired excitation spectra by monitoring the LEI signal. One excitation spectrum for the LEI signal due to the Ga $4^{2} P_{1 / 2} \rightarrow 4^{2} D_{3 / 2}$ transition is presented in Fig. 3 . The dye laser was well tuned to the line center of each considered transition. Then the power dependence of the LEI signal was carried out to ensure that the ratio $\left(S_{u l}\right)$ $I_{\lambda}$ ) would be measured under weak laser intensity. Two power dependence curves of the LEI signal due to the Ga $4^{2} P_{1 / 2} \rightarrow 4^{2} D_{3 / 2}$ and $4^{2} P_{3 / 2} \rightarrow 4^{2} D_{3 / 2}$ transitions are presented in Fig. 4a and 4 b, respectively.

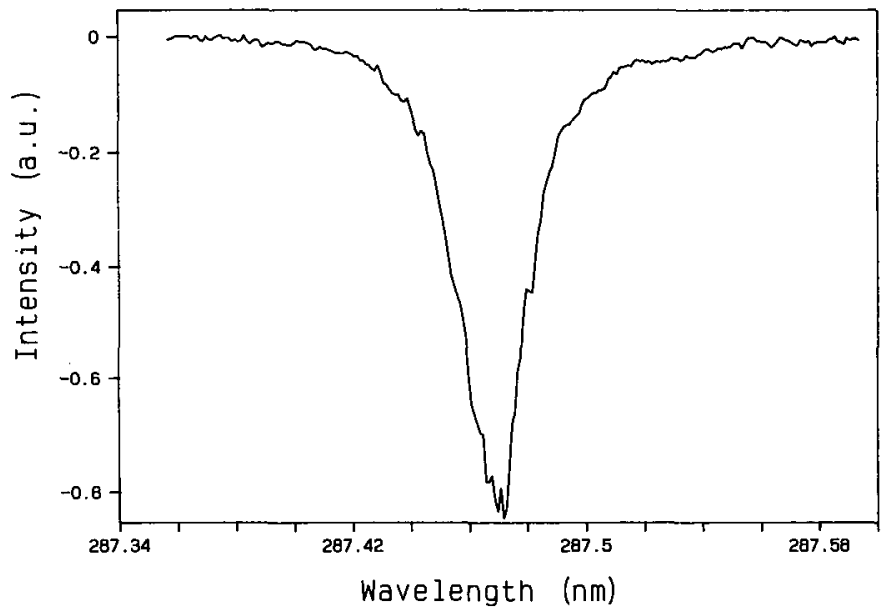

Fig. 3. Excitation spectrum for the laser-enhanced ionization signal due to the Ga $4^{2} P_{1 / 2} \rightarrow 4^{2} D_{3 / 2}$ atomic transition.
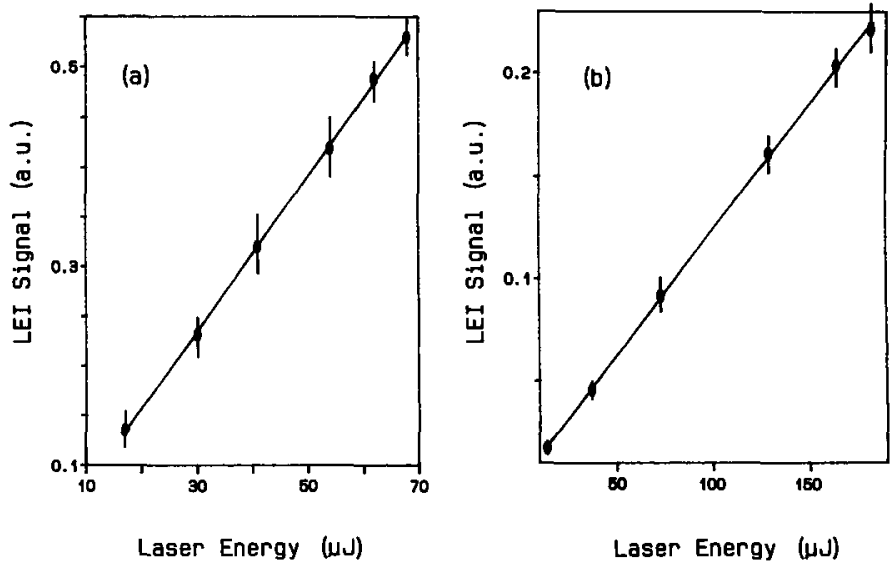

Fig. 4. Power dependence curves for the laser-enhanced ionization signal due to the Ga atomic transitions: (a) $4^{2} P_{1 / 2} \rightarrow 4^{2} D_{3 / 2}$ and (b) $4^{2} P_{3 / 2} \rightarrow 4^{2} D_{3 / 2}$.

\section{RESULTS AND DISCUSSION}

The flame temperature can be determined feasibly and effectively, according to Eq. 10, in which the ratio $S_{u l} / I_{\lambda}$ may be obtained from the measurement of the laser power dependence of the Ga LEI signal. The resultant LEI measurements as a function of the laser power with transition $4^{2} P_{1 / 2} \rightarrow 4^{2} D_{3 / 2}$ or $4^{2} P_{3 / 2} \rightarrow 4^{2} D_{3 / 2}$, are given in the Fig. 4. The energy difference between the fine structures is $827 \mathrm{~cm}^{-1}$, and therefore the upper level can be thermally populated. Note that the laser power was maintained in the regime of under-saturation; that is, the LEI signal of the Ga sample was ensured as being linearly proportional to the laser power. This condition is the criterion for valid application of Eq. 10. In order to confirm that the derived model is applicable to any threelevel system, we performed the LEI measurements with excitation to different intermediate states of $\mathrm{Ga}$ analyte. The values of $2504 \pm 74 \mathrm{~K}$ and $2521 \pm 31 \mathrm{~K}$ were thus obtained for the two selected intermediate states, $5^{2} S_{1 / 2}$ and $4^{2} D_{3 / 2}$, respectively. The excitation transitions and associated transition probabilities are listed in Table I. Accordingly, the excellent agreement under different intermediate states implies success of the three-level model as applied to the flame temperature measurement.

To further justify that the LEI technique is indeed an alternative for monitoring flame temperature, some pertinent measurements previously reported for the same air/acetylene flame are also listed in Table II. The temperature ranges from 2400 to $2600 \mathrm{~K}$ when measured by optical spectroscopy, including atomic absorption (AA), atomic emission (AE), and atomic fluorescence (AF). One can see that our measurements agree very well with those determined by other techniques, ensuring that flame temperature determination using the LEI technique can be as reliable as other well-known optical methods.

Compared to the LEI method ${ }^{15}$ adopted previously with the use of the Einstein relation, the model developed in this work simplifies the task for the purpose of flame temperature determination. To use the Einstein relation, one must measure the ion diffusion and ion mobility coefficients. It is not trivial, however, to take a precise measurement of these coefficients without making the following assumptions or simplification. First, the elec- 
TABLE II. Flame temperature of an atmospheric air/acetylene flame determined by using the LEI method and other techniques. AA, AE, and $\mathrm{AF}$ in the first column represent atomic absorption spectroscopy, atomic emission spectroscopy, and atomic fluorescence spectroscopy, respectively. The flow rates (in $L / \mathrm{min}$ ) of air and fuel, denoted as $\mathrm{A} / \mathrm{F}$, and the measured position (in $\mathrm{mm}$ ), denoted as $\mathrm{H}$, from the burner head are separately listed in the second and third columns.

\begin{tabular}{ccclc}
\hline $\begin{array}{c}\text { Meth- } \\
\text { od }\end{array}$ & A/F & \multicolumn{1}{c}{ H } & \multicolumn{1}{c}{ Temperature (K) } & Reference \\
\hline LEI & $12.5 / 0.5$ & 7.5 & $2504 \pm 74,2521 \pm 31$ & This work \\
AA & $9.44 / 0.96$ & 5.0 & 2450 & 18 \\
AE & $9.44 / 0.96$ & 5.0 & 2460,2480 & 18 \\
AE & $10.0 / 1.35$ & $1.5(4.5)$ & $2430 \pm 35(2420 \pm 25)$ & 19 \\
AF & $9.8 / 1.5$ & 2.0 & $2540 \pm 25$ & 20 \\
AA & $9.5 / 1.3$ & 4.5 & 2452,2473 & 21 \\
AF & $8.25 / 1.15$ & 32.0 & $2545 \pm 60,2555 \pm 50$ & 22 \\
AF & $7.6 / 1.04$ & $3(48)$ & $2400(2600)$ & 23 \\
\hline
\end{tabular}

tric field must be assumed to be constant, as a result of neglect of the shielding effect of space charge, caused by the intense charge density either from the species of interest or from the thermal ions. Second, the convection velocity must be neglected to simplify the continuity equation. And finally, the size of the electrode must be assumed to be dimensionless, when one is measuring the distance from the ion source to the electrode. The resultant data propagation no doubt leads to a large systematic error.

In contrast, the version of LEI method described here appears to be more convenient and precise; only two approximations-a steady-state approximation for the intermediate state and the Boltzmann distribution for the population of fine structures-are assumed. It is reasonable to use a steady-state approximation since the excitation rate, limited to the condition of under-saturation, is small, and the ionization rate for the excited atoms is relatively larger since the energy defect from the intermediate state to the ionization continuum becomes smaller. As soon as the intermediate state is populated, it can be depleted rapidly. This suggests that a steady-state approximation should be valid for the system. ${ }^{6,16,24}$ On the other hand, the Boltzmann distribution is applicable to a local thermal equilibrium flame, which has been assumed in most of methods. ${ }^{15,25}$

In summary, we have both theoretically and experimentally demonstrated that the LEI technique can be a simple, alternative way to determine flame temperature if an appropriate element is selected as analyte, provided that its two lowest atomic levels are close enough and that transition probabilities to one common upper level are also given. We have used the Ga analyte for the LEI test with excitation to various intermediate states. Our measurements are consistent with each other and also agree very well with previously reported results.

\section{ACKNOWLEDGMENT}

This work is supported in part by the National Science Council of the Republic of China. We thank Mr. Ching-Bin Ke for his helpful assistance during the experiments.

1. C. Th. J. Alkemade, T. Hollander, W. Snelleman, and P. J. Th. Zeegers, Metal Vapours in Flames (Pergamon Press, New York, 1982).

2. D. R. Crosley, Laser Probes for Combustion Chemistry, ACS Symp. Ser. No. 134 (American Chemical Society, Washington, D.C., 1980).

3. J. C. Travis, G. C. Turk, and R. B. Green, Anal. Chem. 54, 1006A (1982).

4. C. A. van Dijk, F. M. Curran, K. C. Lin, and S. R. Crouch, Anal. Chem. 53, 1275 (1981).

5. O. Axner, IU. Magnusson, J. Peterrson, and S. Sjostrom, Appl. Spectrosc. 41, 19 (1987).

6. N. Omenetto, Th. Berthoud, P. Cavalli, and G. Rossi, Anal. Chem. 57, 1256 (1985).

7. O. Axner, I. Lindgren, I. Magnusson, and H. Rubinsztein-Dunlop, Anal. Chem. 57, 773 (1985).

8. K. C. Lin and Y. S. Duh, Appl. Spectrosc. 43, 20 (1989).

9. G. C. Turk, J. R. DeVoe, and J. C. Travis, Anal. Chem. 54, 643 (1982).

10. T. Berglind, S. Nillson, and H. Rubinstein-Dunlop, Phys. Scripta 36, 246 (1987).

11. K. S. Epler and T. C. O'Haver, Anal. Chem. 60, 2062 (1988).

12. B. W. Smith, L. P. Hart, and N. Omenetto, Anal. Chem. 58, 2147 (1986).

13. T. A. Cool, Appl. Optics 23, 1559 (1984).

14. E. G. Mallard and K. C. Smyth, Comb. Flame 44, 61 (1982).

15. K. C. Lin, P. M. Hunt, and S. R. Crouch, Chem. Phys. Lett. 90, 111 (1982).

16. J. C. Travis, J. Chem. Educ. 59, 909 (1982).

17. W. L. Wiese and G. A. Martin, in Wavelengths and Transition Probabilities for Atoms and Atom Ions, Part II, NSRDS-NBS 68 (1980).

18. L. De Galan and G. F. Samaey, Spectrochim. Acta 25B, 245 (1972).

19. G. F. Kirkbright, M. Sargent, and S. Vetler, Spectrochim. Acta 25B, 465 (1970).

20. M. Omenntto, D. Benetti, and G. Rossi, Spectrochim. Acta 27B, 453 (1972).

21. R. F. Browner and J. D. Winefordner, Anal. Chem. 44, 247 (1972).

22. H. Haraguchi, B. Smith, S. Weeks, D. J. Johnson, and J. D. Winefordner, Appl. Spectrosc. 31, 156 (1977).

23. H. Haraguchi and J. D. Winefordner, Appl. Spectrosc. 31, 195 (1977).

24. L. Volk, W. Richardson, K. H. Lau, M. Hall, and S. H. Lin, J. Chem. Educ. 54, 95 (1977).

25. A. G. Gaydon and H. G. Wolfhand, Flames: Their Structure, Radiation and Temperature (Chapman and Hall, London, 1979), 4th ed. 